\title{
Selection of NIR wavelengths from hyperspectral imaging data for the quality evaluation of Acerola fruit
}

\author{
Cristina Malegori ${ }^{1}$, Aoife Gowen ${ }^{2}$, Emanuel Marques ${ }^{3}$, Maria Fernanda Pimentel Avelar ${ }^{4}$, Sergio \\ Freitas $^{5}$, Celio Pasquini $i^{6}$, Ernestina Casiraghi ${ }^{7}$ \\ ${ }^{1}$ Department of Food Environmental and Nutritional Science - Università degli Studi di Milano \\ ${ }^{2}$ University College Dublin - Biosystems Engineering - School of Agriculture, Food Science and \\ Veterinary Medicine \\ ${ }^{3}$ Universidade Federalde de Pernambuco - Departamento de Engenharia Química \\ ${ }^{4}$ Universidade Federal de Pernambuco, Departamento de Engenharia Química \\ ${ }^{5}$ Bolsista produtividade CNPq / EMBRAPA - Embrapa Semiárido - Fisiologia e Tecnologia Pós- \\ colheita / EMBRAPA \\ ${ }^{6}$ Chemistry Institute - UNICAMP - Departmente of Analytical Chemistry \\ ${ }^{7}$ University of Milan - Faculty of Agricultural and Food Science - DeFENS
}

Hyperspectral imaging is an alternative technique for non-destructive food analysis, enabling real-time monitoring of quality. Each pixel of a hyperspectral image contains the spectrum of that specific position thus obtaining both spatial and spectra information of a sample. The data are three-dimensional blocks, called hypercubes, analysed with a chemometric approach that reduces dimensionality of the data while retaining the most useful spectral information. Therefore, hyperspectral imaging is a powerful tool for the identification of the key wavelengths in the development of multispectral device, intended for specific product control. The aim of this work is to evaluate Acerola post-harvest quality using NIR hyperspectral imaging.

Hyperspectral images of 20 acerolas were acquired for 5 consecutive days, giving a total of 100 images. The fruits were characterized by different initial ripeness degree, evaluated by the colour according with current market methods, and were stored at room temperature $\left(25 \pm 2{ }^{\circ} \mathrm{C}\right)$. A SisuChema Hyperspectral Camera $(900-2500 \mathrm{~nm}$ ) was used, equipped with a $50 \mathrm{~mm}$ lens with a minimum resolution of $150 \mu \mathrm{m}$. Using the spectral data, an investigation of trends along time was carried out to highlight the most important three wavelengths that characterized the ripeness/degradation process of the Acerola fruit. The mean spectra of each fruit at day 1 was calculated and subtracted to all the images of the corresponding fruits, at the other four sampling days. The subtracted images were refolded in new hypercubes, allowing the visualization of a grayscale map of each fruit at the three interesting wavelengths. The three grayscale images were combined into a false RGB image for a global evaluation along time.

The three interesting wavelengths selected were $1883 \mathrm{~nm}$, due to the second overtone of the $\mathrm{C}=\mathrm{O}$ bond, $1407 \mathrm{~nm}$, the absorption band of the $\mathrm{CH}$ first overtone combination and first $\mathrm{OH}$ overtone, and $1136 \mathrm{~nm}$ characterized by the second overtone of $\mathrm{CH}$ bond. A little variability of the acerola pre-processed NIR profile is evident during time. Therefore, a colour change from green to red, which is considered as a ripeness evolution index, does not point out a modification of the fruit composition, commonly reflected in NIR spectra. This is confirmed by the histogram profiles (grey scale intensity vs frequency) of each false image at the three selected wavelengths. Spectra modification, that allowed variable selection, appears more evident when acerola colour turns from red to purple-brown describing the senescence process of the fruit. 
Acerola appears to be a non-climacteric fruit, meaning that ripening stops after harvest. The false-colour RGB images derived from the hyperspectral data enable early detection of the senescence process in a rapid and non-destructive manner. Ultimately, these results could be used to develop a low cost multispectral imaging system for acerola shelf-life evaluation. 